Garita Cordero, M., Rojas Sáenz, M.J. y Rojas Sancho, M. (2022). PATRIMONIO ARQUEOLÓGICO DE CACHÍ: UN PROYECTO VIRTUAL DE ARQUEOLOGÍA PARA LA COMUNIDAD EN EL CONTEXTO DEL COVID-19. Revista Herencia, 35(1), enero-junio, 100-108.

\title{
PATRIMONIO ARQUEOLÓGICO DE CACHÍ: UN PROYECTO VIRTUAL DE ARQUEOLOGÍA PARA LA COMUNIDAD EN EL CONTEXTO DEL COVID-19
}

Cachí’s Archeological Heritage: A Virtual Project of Community Archeology in the Context of Covid 19

Recibido: 28-10-2021

Aprobado: 23-12-2021

María José Garita Cordero, Bachiller en Antropología de la Universidad de Costa Rica. Actualmente estudia ingeniería en informática en la Universidad Nacional de Educación a Distancia.

María Jesús Rojas Sáenz, es Bachiller en Antropología de la Universidad de Costa Rica. Actualmente, estudia la Licenciatura de Arqueología y el Bachillerato de Turismo Ecológico en la Universidad de Costa Rica.

María de los Ángeles Rojas Sancho, Bachiller en Antropología de la Universidad de Costa Rica y estudiante de la maestría en Antropología. Actualmente labora en el Museo Nacional de Costa Rica en el Departamento de Antropología e Historia.
María Garita Cordero

Universidad de Costa Rica, Costa Rica maria.garitacordero@ucr.ac.cr

María Jesús Rojas Sáenz

Universidad de Costa Rica, Costa Rica maria.rojassaenz@ucr.ac.cr

María de los Ángeles Rojas Sancho Museo Nacional de Costa Rica, Costa Rica mrojass@museocostarica.go.cr

\section{RESUMEN}

El proyecto de Patrimonio Arqueológico de Cachí (PADC) surge en el año 2020 en respuesta a una serie de acciones de destrucción y huaquerismo hacia el patrimonio arqueológico local. El presente proyecto fue de carácter virtual debido a las limitaciones de la pandemia del Covid-19 y tuvo como objetivo promover la concientización y vinculación del patrimonio arqueológico en la comunidad; esto se logró a través de la creación de recursos virtuales que facilitaron la socialización de los yacimientos y su valor histórico. Para ello, se creó una página de Facebook e Instagram, para realizar publicaciones informativas. Posteriormente, con el apoyo financiero del programa de Becas Creativas del Ministerio de Cultura y Juventud se crearon materiales divulgativos como: podcasts, audiogramas y conversatorios virtuales con la comunidad. Lo que promovió una respuesta activa de las personas con las páginas de Facebook e Instagram, que se tradujo en el rescate de la memoria de los habitantes en torno al patrimonio. A modo de conclusión, se recalca la importancia de involucrar a las comunidades en la socialización del valor del patrimonio y acciones de conservación, especialmente en tiempos donde el uso de tecnologías es cotidiano.

Palabras clave: Arqueología; virtual; patrimonio; petrograbados; Covid-19

\footnotetext{
ABSTRACT

Cachí's Archeological Heritage emerged in 2020 as a response to a series of actions of destruction towards the archaeological heritage of the Cachí district. Also, the objective of the project was to promote awareness and linkage of archaeological heritage in the Cachí community through the creation of virtual resources that will facilitate the socialization of archaeological sites and their historical value. Therefore, we created Facebook and Instagram pages to make informative publications. Then, the project won a scholarship from the Ministry of Culture and Youth, which allowed the creation of various informative materials like: podcasts, audiograms and virtual conversations with the community. Which promoted an active response from people with Facebook and Instagram pages, which resulted in the rescue of the community's memory around heritage. In conclusion, the importance of involving communities in the socialization of heritage value and conservation actions is emphasized, especially in times where the use of technologies is daily.
}

Keywords: Archeology; virtual; heritage; petroglyphs, Covid-19 
Revista Herencia, Vol. 35 (1), enero-junio, 2022.

\section{Introducción}

El proyecto de Patrimonio Arqueológico de Cachí fue realizado en la provincia de Cartago, en el distrito de Cachí del cantón de Paraíso, en un lapso de nueve meses desde marzo hasta noviembre del año 2020. Surgió como respuesta a una serie de acciones destructivas hacia el patrimonio arqueológico local, las cuales consistieron en movimientos de tierra que dejaron al descubierto los yacimientos arqueológicos, el posterior saqueo a estos yacimientos y el deterioro de los petrograbados por factores antrópicos (ver figura 1).

Los petrograbados son relevantes en Cachí ya que se han ubicado varios en la zona, Monge (2021) los describe como rocas que presentan diseños creados durante la época precolombina por las poblaciones indígenas. Para realizarlos se emplearon herramientas como percutores y cinceles, los cuales permitían realizar desgastes e incisiones en las rocas. En la actualidad los petrograbados están protegidos por la Ley 6703 de Patrimonio Nacional Arqueológico y son parte del patrimonio de Costa Rica.

Figura 1. Petrograbado afectado por saqueo.

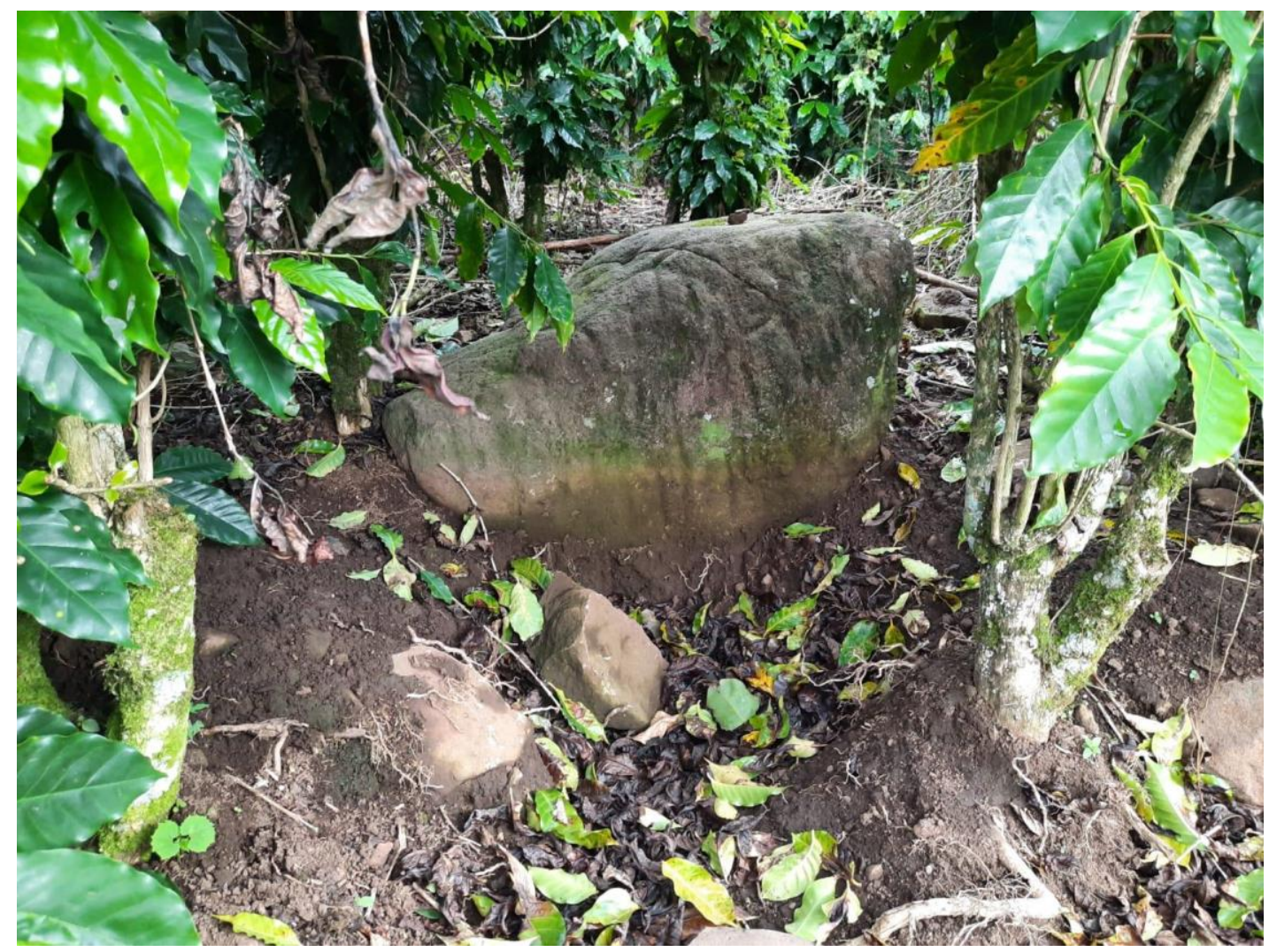

En la fotografía se aprecia el levantamiento de tierra al lado de un petrograbado, vinculado a acciones de saqueo. Fuente: Elaboración propia.

El objetivo del proyecto fue promover la concientización y vinculación del patrimonio arqueológico en la comunidad de Cachí a través de la creación de recursos virtuales que 
Revista Herencia, Vol. 35 (1), enero-junio, 2022.

facilitaran la socialización de los sitios arqueológicos y su valor histórico. Estaba dirigido específicamente al distrito de Cachí, debido a que se identificaron que quienes estaban dañando el patrimonio arqueológico local eran personas de la misma comunidad; las cuales vieron afectadas sus fuentes de ingresos económicos a partir de las medidas sanitarias tomadas por Costa Rica por la pandemia del Covid-19.

La economía de Cachí se concentra en actividades agropecuarias, pequeños comercios, turismo y venta de artesanías, entre otras (Araya, Arias y Cerdas, 2002), por lo cual al darse los cierres de comercios y de actividades turísticas a partir de las medidas sanitarias, los ingresos económicos de las personas locales se vieron afectados durante los primeros meses del año 2020. Lo anterior puso en vulnerabilidad económica a este sector de la población que no tenía otras opciones para solventar la crisis.

Asimismo, la crisis económica acrecentó la destrucción del patrimonio en general, según lo reporta la UNESCO (2020), la organización relacionó directamente el contexto socioeconómico afectado por la pandemia del Covid-19 con el saqueo al patrimonio natural y cultural mundial por parte de las personas. Por lo tanto, lo observado en la comunidad de Cachí responde a la necesidad de las personas por encontrar alternativas económicas a una situación extrema que conllevó al saqueo del patrimonio arqueológico y parte de su destrucción.

\section{Metodología}

En respuesta a los saqueos de los sitios arqueológicos y destrucción de los petrograbados e inmersos en el contexto de cierres de comercios, instituciones públicas y actividades en el país debido a la pandemia por COVID-19, se tomó la iniciativa de trabajar de manera virtual, llevando a cabo acciones a corto plazo de aproximadamente 3 meses y a largo plazo 6 meses, con el propósito de concientizar a la población de la comunidad de Cachí sobre la importancia de su historia antigua.

Ahora bien, entre las acciones a corto plazo, se crearon dos páginas del proyecto Patrimonio Arqueológico de Cachí en Facebook e Instagram y en estas se compartieron publicaciones e información relacionada sobre la protección e importancia del patrimonio arqueológico local. En cuanto a las acciones a largo plazo, se propuso la creación de diferentes materiales audiovisuales, tales como: un podcast, audiogramas y conversatorios con personas de la comunidad de Cachí.

El proyecto tuvo dos etapas metodológicas; la primera fueron las acciones a corto plazo, en el que el eje principal fue la creación de una página de Facebook e Instagram como medio de comunicación con la comunidad de Cachí. De este modo, realizar arqueología para la comunidad de manera virtual no solo fue un reto, sino que también fue una oportunidad debido a que las redes sociales han transformado el acceso a la cultura y el arte por parte de las personas, la cual permite realizar difusión a un público más extenso (Costa, 2017).

En la página se compartió información sobre la conservación del patrimonio arqueológico e historia antigua, en donde se abarcaron temas en relación con los petrograbados, tales 
Revista Herencia, Vol. 35 (1), enero-junio, 2022.

como: qué son, cómo se hicieron y cómo se protegen. Se utilizó un lenguaje simple y claro sin uso de tecnicismos científicos (ver Figura 2) para que este fuese más accesible y de mayor comprensión para el público general de la localidad.

Figura 2. Afiche informativo sobre el cuidado de los Petrograbados.
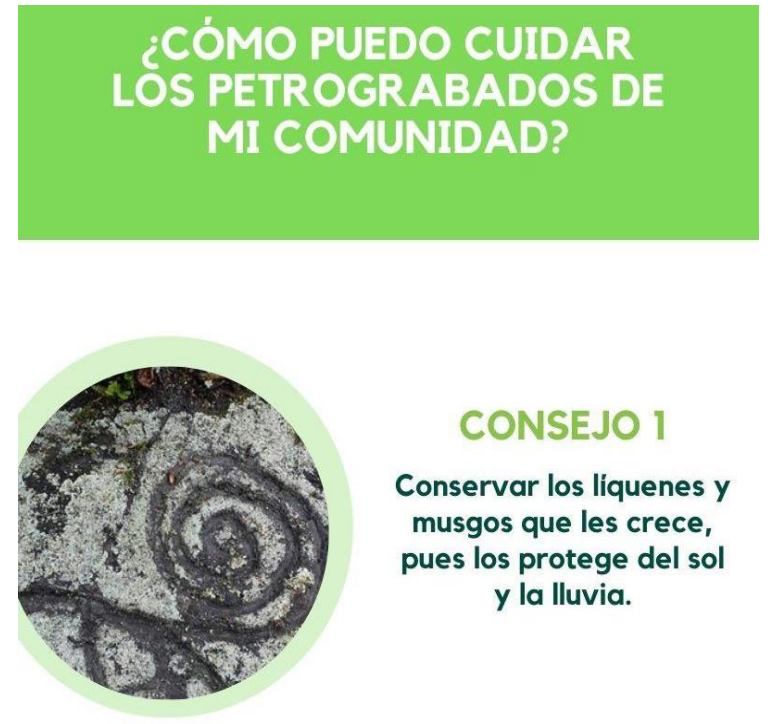

\section{CONSEJO 1 \\ Conservar los líquenes y musgos que les crece, pues los protege del sol y la lluvia.}

CONSEJO 2

Dejarlos en los lugares donde aparecieron, esto permite estudiarlos en un futuro.


\section{CONSEJO 3 \\ Evitar rayarlos con tiza o pintura porque los daña.}

Fuente: Elaboración propia.

Con respecto de la segunda etapa, el proyecto recibió apoyo financiero por parte del Ministerio de Cultura y Juventud con el programa "Becas Creativas", lo cual facilitó ampliar el rango de actividades, dentro de estas se detallan: conversatorios, audiogramas y un podcast. En cuanto a los conversatorios: radicaron en crear espacios virtuales de diálogo para compartir experiencias, anécdotas e historias relacionadas con el patrimonio 
Revista Herencia, Vol. 35 (1), enero-junio, 2022.

arqueológico de la zona de Cachí. Esto tuvo como objetivo la identificación de la forma en que las personas se vinculan con el patrimonio y cuáles eran sus pensamientos, interpretaciones y sentires sobre este. En total se realizaron cuatro conversatorios en los cuales participaron seis personas de la comunidad las cuales lo hicieron de forma anónima.

La actividad que tuvo mayor complejidad fue el podcast, llamado "Grabados en Nuestra Memoria" porque fueron episodios de audio de treinta minutos a una hora, los cuales fueron publicados por medio de Anchor en varias plataformas como Spotify, Podcast de Apple, Podcast de Google, Breaker, Pocket Cast, entre otros. Este contó con tres episodios diferentes: 1) Nuestro Origen, 2) Significados e identidad y 3) Relatos y Memorias de Cachí. Asimismo, se incluyó una sección denominada "La evidencia de la semana", de la cual se produjeron también tres ediciones: 1) Las puntas paleoindias, 2) Sociedades que navegaron y 3) Escultura tipo Chac mool.

En cuanto al contenido de cada uno, el primer podcast fue titulado "Nuestro origen" y en este se invitó a participar a la arqueóloga Mónica Aguilar con el fin de conversar sobre la historia antigua y diversidad cultural del país. En el segundo episodio "Significados e Identidad" se invitó al arqueólogo Francisco Corrales y en esta ocasión se habló de las múltiples formas en las que las comunidades se apropian, resignifican y entienden su propio patrimonio arqueológico y herencia ancestral. Finalmente, en el tercer podcast "Relatos y Memorias de Cachí", se platicó con varias familias de la zona sobre las diferentes experiencias que han tenido con el patrimonio arqueológico.

En lo que respecta a la sección de la "Evidencia de la semana", en la primera publicación participó el arqueólogo Abraham Zúñiga del Museo Nacional de Costa Rica y se trajo a colación el tema de poblamiento en el continente americano, específicamente el territorio que actualmente es Costa Rica y las diferentes evidencias que estos grupos dejaron. En la segunda edición "Sociedades que navegaron" se tuvo como invitada a la arqueóloga Yajaira Núñez Cortés, con quien se compartió sobre las evidencias asociadas a la navegación en el pasado. Finalmente, la tercera se denominó "Escultura tipo Chacmool" y esta vez el invitado fue el arqueólogo Ricardo Vázquez, con quien se charló sobre el Monumento Arqueológico Las Mercedes.

Asimismo, los audiogramas, constaron de una sección de audio de corta duración derivados de las reflexiones de los participantes tanto del podcast cómo de los conversatorios. Este punto es de suma importancia porque favoreció el diálogo horizontal y, además, se visibilizan las perspectivas, saberes y opiniones de la comunidad.

Cabe mencionar que tanto estas dos etapas como las estrategias metodológicas implementadas se plantearon con el propósito de revitalizar las memorias sobre los hechos y eventos históricos que han sido puestos en un segundo plano u olvidados con el paso del tiempo y las nuevas generaciones; al mismo tiempo estas iniciativas buscaron fomentar la identidad relacionada con los grupos culturales que poblaron el actual territorio de Cachí.

En primer lugar, los materiales educativos publicados en redes sociales y los conversatorios facilitaron un contacto más cercano con las personas de la comunidad, ya que, a partir de 
Revista Herencia, Vol. 35 (1), enero-junio, 2022.

estos se logró interactuar con los habitantes de la zona. Esto permitió diagnosticar el vínculo y sentires de las y los vecinos con respecto de los bienes patrimoniales. En segundo lugar, los conversatorios plasmaron parte de las vivencias y experiencias de las personas durante su niñez y juventud con los elementos arqueológicos circundantes. A raíz de lo anterior, es posible concluir lo siguiente:

a) La población que demostró interés en el tema y en participar de las actividades desarrolladas perteneció a un grupo etario que estuvo compuesto mayormente por adultos. En relación con lo anterior, en los conversatorios participaron un total de 6 personas, en donde la mayoría tenía entre 30 a 59 años. (Ver gráfico 1).

Cabe mencionar que estos datos corresponden únicamente a los participantes de los conversatorios, ya que solo con estas personas fue posible trabajar inferencias cualitativas y cuantitativas, sin embargo, el público de las páginas de redes sociales era más amplio, sobrepasando los 1000 seguidores.

Gráfico 1. Rango de las edades de los participantes según género.

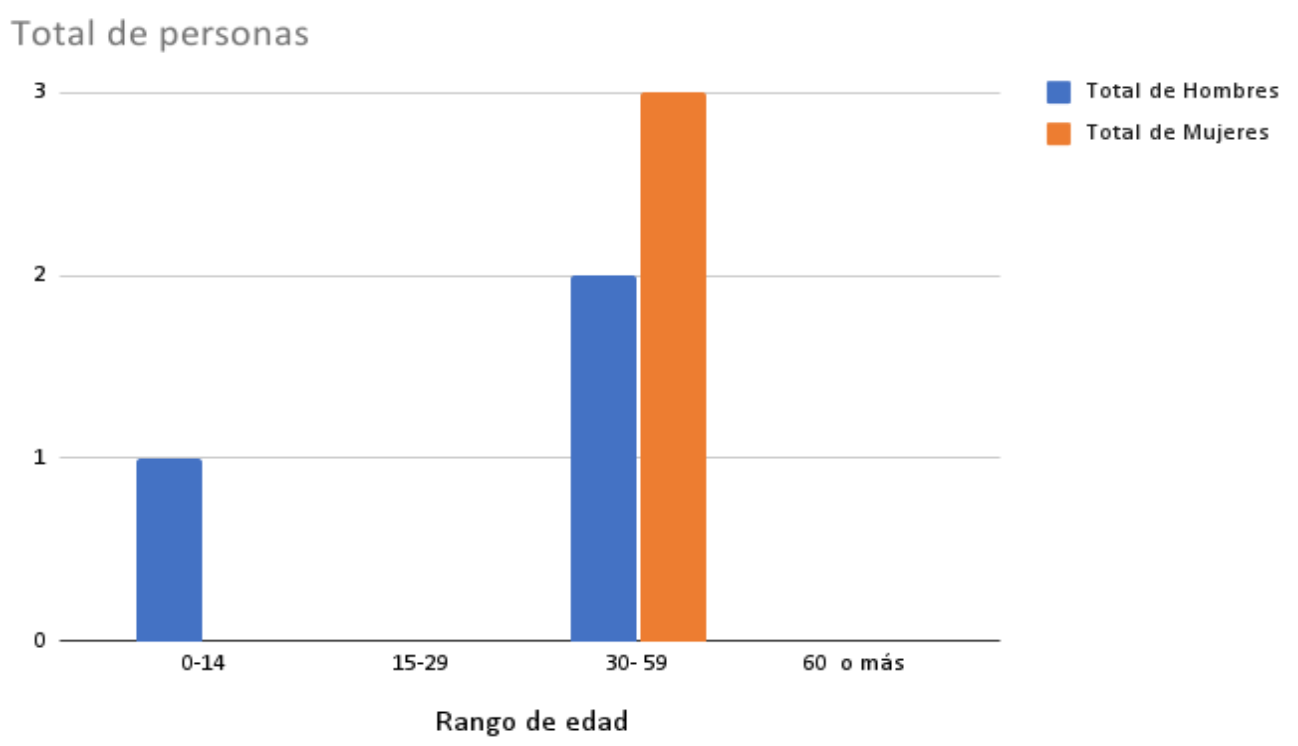

Fuente: Elaboración propia.

b) El patrimonio no es un tema ajeno para la población local, ya que, a partir de las vivencias relacionadas con elementos arqueológicos, la comunidad creó sus propias interpretaciones y significados sobre los grupos que habitaron anteriormente este territorio.

c) De los seis participantes de los conversatorios, el total de adultos entre el rango de 30 y 59 años, afirmaron tener un vínculo emocional con el patrimonio arqueológico local justificado desde la memoria y el recuerdo de distintas vivencias relacionadas, tanto con objetos arqueológicos, como con los espacios geográficos en donde se localizan los yacimientos. En ligamen con lo anterior, una de las causas de este fenómeno puede deberse a sus experiencias cómo recolectores de café o cuando de niños jugaban en el cafetal. 
Revista Herencia, Vol. 35 (1), enero-junio, 2022.

A manera de ejemplificación de lo anterior, por un lado, en los conversatorios varias personas comentaron sobre las familias de los Murray y los Lindo, las cuales tuvieron gran relevancia en el desarrollo económico local ya que eran propietarios de la mayoría de los cafetales y beneficios cafetaleros en donde trabajaba la población de la zona.

Por el otro lado, una de las participantes de los conversatorios expresó su afecto por uno de los petrograbados de Cachí al comentar que de pequeña solía frecuentar un petrograbado en los días de verano, ya que, al salir de clases se iba con sus compañeros a bañarse al río; cabe mencionar que este hecho creó múltiples recuerdos que la participante guarda con cariño hasta hoy.

En lo que respecta a la memoria y al recuerdo es posible concluir que tanto el espacio geográfico, como las vivencias de una generación han influido en la construcción de una identidad local; es decir, uno de los participantes afirmó que muchos de los pobladores actuales, como sus padres y abuelos, habían trabajado en esta zona como recolectores de café y debido a esto crearon un vínculo con los yacimientos arqueológicos. Además, este participante menciona que para las generaciones más recientes este sentimiento ha ido desapareciendo debido a que los jóvenes locales han comenzado a dedicarse a otras prácticas económicas; por lo tanto, el participante concluye que para no perder el vínculo con sus antepasados e historia antigua, lleva a sus hijas y a sus sobrinas a visitar los petrograbados de la zona de Cachí.

En cuanto a lo que respecta al tema de conservación, todas las personas participantes de los conversatorios manifestaron inquietudes y preocupaciones vinculadas con la destrucción y deterioro de sitios arqueológicos y el patrimonio de su comunidad. Por ejemplo, una de las participantes hizo hincapié en la importancia de ubicar los sitios arqueológicos de la zona para su correcta conservación, ya que ella piensa que la mayoría de los sitios han sido destruidos por la construcción de infraestructura o casas. Además, esta persona mencionó que Cachí es una oportunidad para entender y proteger el patrimonio ya que esta zona aún se dedica al café y la agricultura por lo que parte de su patrimonio aún no ha sido destruido.

Por otra parte, a nivel nacional la producción de material informativo destinado a un público no académico es reducido. Por lo tanto, los podcast, infografías y audiogramas representaron la iniciativa de generar herramientas que tienen la ventaja de permanecer para la consulta posterior con temas relacionados con historia antigua, arqueología y patrimonio desde el enfoque educativo y del entretenimiento.

Asimismo, por medio del podcast y de su complemento llamado "La evidencia de la semana" fue posible explicar sobre eventos que acontecieron en el pasado por medio de los bienes arqueológicos en cuestión. De esta manera, se pudo trascender de la apreciación estética de las piezas arqueológicas hacia la valoración histórica.

Finalmente, los distintos esfuerzos y presencia en redes sociales influyeron en la declaratoria de dos monumentos arqueológicos en esta localidad en coordinación con el 
Revista Herencia, Vol. 35 (1), enero-junio, 2022.

Museo Nacional de Costa Rica, los cuales fueron: Agapanto (C-481 Ag) y Orus (C-482 Or) (Solís, 2020).

\section{Conclusiones}

Se destaca la importancia de involucrar a las comunidades para compartir, informar y guiar sobre la historia antigua, la socialización de su valor y conservación. En consecuencia, el desconocimiento de las personas de Cachí sobre su propia historia ancestral da como resultado un efecto contrario a la conservación, debido a que se imposibilita a los actores más cercanos influir en los procesos de salvaguarda.

Frente al aumento en recortes de presupuesto de muchas instituciones culturales, la conservación de los sitios arqueológicos se ha visto afectada, en este sentido, se recomienda involucrar a la comunidad mediante una adecuada comunicación y en el uso de herramientas informativas. Esto puede generar que las personas se conviertan en actores claves en la protección de su patrimonio local, si la comunidad conoce el valor de su historia antigua, lo compartirá con las futuras generaciones, evitando prácticas invasivas y denunciando acciones de personas que atenten contra la conservación del patrimonio.

Una de las dificultades enfrentadas por el proyecto de Patrimonio Arqueológico de Cachí en las páginas de redes sociales de Facebook e Instagram fue conectar con un público más joven, aunque esta es la población que domina el uso de redes sociales, no posee un vínculo emocional fuerte con los sitios arqueológicos locales, a diferencia de sus familiares de mayor edad. Esto se debe a que los modos de vida han cambiado y los jóvenes se relacionan menos con los espacios agrícolas y prácticas al aire libre.

Finalmente, la experiencia con la gestión y difusión a través de medios virtuales debido a la pandemia por COVID-19, produjo que el proyecto debiera reinventarse en la búsqueda de nuevas formas de interactuar y acercar los bienes arqueológicos con la comunidad. Especialmente en tiempos donde el uso de tecnologías es cotidiano y la información es más accesible en comparación a unas décadas atrás.

Para la consecución de futuros esfuerzos en materia de socialización del patrimonio arqueológico con comunidades, se proponen las siguientes recomendaciones:

1. Aplicar un diagnóstico previo a la creación de las páginas en redes sociales con el fin de identificar actores claves, amenazas y público meta. A partir de este, se pueden desarrollar estrategias concretas que respondan al contexto de cada comunidad.

2. El tema de la conservación de sitios arqueológicos dirigido a las comunidades se encuentra en desarrollos iniciales en el país por lo que se invita a que se exploren ampliamente estrategias y herramientas que permitan a las comunidades conocer cómo proteger su patrimonio.

3. Se insta a apoyar las iniciativas locales de enseñanza y conservación, de manera que los investigadores establezcan un rol de mediador, más allá de un papel de autoridad. La 
Revista Herencia, Vol. 35 (1), enero-junio, 2022.

clave para esto es el diálogo horizontal entre los conocimientos científicos de los arqueólogos y arqueólogas con las interpretaciones del patrimonio de las personas locales.

\section{BIBLIOGRAFÍA}

Araya, M., Arias, C. y Cerdas, L. (2002). "Si no unimos lo que tenemos, nadie va ha hacerlo... Gestión local para la reducción del riesgo ante los desastres en Cachí, Paraíso, Cartago”. [Tesis de Licenciatura, Universidad de Costa Rica].

Corrales, F. (2019). Hacia una arqueología pública en el Sureste de Costa Rica: tres experiencias. I Simposio de Arqueología Pública en El Salvador.

Costa, T. (2017). Instagram como herramienta para la creación de un museo social y online. [Tesis de Maestría, Universidad Autónoma de Barcelona].

Ministerio de Cultura y Juventud. (2020). Becas Creativas.

Monge, G. (7 de septiembre de 2021). Petrograbados en el Río General de Pérez Zeledón. Museo Nacional de Costa Rica. https://www.museocostarica.go.cr/divulgacion/articuloseducativos/petrograbados-en-el-rio-general/

Solís, F. (2020). Informe de inspección arqueológica por denuncia de alteración de sitios arqueológicos en Cachí, Paraíso, Cartago. Museo Nacional de Costa Rica. Departamento de Antropología e Historia.

UNESCO. (2020). Cultura y Covid-19. Impacto y Respuesta. 
Revista Herencia, Vol. 35 (1), enero-junio, 2022. 